\title{
Determination of Changes in Sulphur Oxidation States in Prostate Cancer Cells
}

\author{
J. Czapla ${ }^{a}$, W.M. Kwiatek ${ }^{a *}$, J. Lekki $^{a}$, R. Steininger ${ }^{b}$ And J. Götthicher $^{b}$ \\ ${ }^{a}$ The Henryk Niewodniczański Institute of Nuclear Physics, Polish Academy of Sciences \\ E. Radzikowskiego 152, 31-342 Kraków, Poland \\ ${ }^{b}$ Karlsruhe Institute of Technology, Institute for Synchrotron Radiation \\ Hermann-von-Helmholtz-Platz 1, D-76344 Eggenstein-Leopoldshafen, Germany
}

\begin{abstract}
Prostate cancer cell lines along with selected organic and inorganic compounds used as references were studied with sulphur $K$-edge X-ray absorption near edge structure spectroscopy. The experiment was performed at the SUL-X beamline of the synchrotron radiation source ANKA, Karlsruhe (Germany). The sulphur was chosen for the studies because it is an essential biological element and out of many relevant factors, it is believed that it can take an important part in cancer transformations. The main goal was to determine which sulphur forms occur in prostate cancer cells and to compare these results with the ones obtained for non-cancerous cells. Therefore oxidation state of this element was analysed with $\mathrm{S} K$-edge X-ray absorption near edge structure spectroscopy. The analysis of $K$-edge structure was done in order to investigate also the chemical structure of the elements neighbouring the central atom. The preliminary results from sulphur X-ray absorption near edge structure in prostate cancer cell line PC-3 and prostatic epithelial cell line PZ-HPV-7 (which was used as a control) show that there are various oxidation states of sulphur occurring in cells. The set of reference compounds with various sulphur oxidation states was used to establish the relation between the energy of the white line maximum and the oxidation state of sulphur. The equation of linear fit was used to compute the unknown oxidation state. In order to obtain a more detailed information the method of deconvolution of X-ray absorption near edge structure spectra was used. Experimental spectra were fitted with two Gaussian peaks and one arctangent step function. Fitting procedure was performed in Athena code and the deconvolution was used to assign the fraction of each sulphur form. The next step was to compare the results calculated for cancerous and non-cancerous cells. In this work, the first results of these studies are presented.
\end{abstract}

PACS: 87.64.kd, 87.19.xj

\section{Introduction}

Although in many countries the mortality rate of prostate cancer has started to fall, the incidence rate is rising quickly. While aggressive testing with prostate specific antigen (PSA) has contributed to this decline in mortality, it does not explain all the effect, because elevated PSA levels may be present in non-malignant conditions such as benign prostatic hyperplasia (BPH). Despite significant advances in cancer diagnostic and research, treatment of high-grade prostate tumours is still palliative $[1,2]$. Therefore every research giving another piece of information about aetiology of this disease is needed and important.

The use of X-ray absorption near edge structure (XANES) spectroscopy can reveal the type and number of ligands, distances and geometry along with the oxidation states of metals and other trace elements in

\footnotetext{
* corresponding author; e-mail: Wojciech.Kwiatek@ifj.edu.pl
}

biological samples. It can be applied as a "standalone" technique, but it is particularly powerful when used with other X-ray and spectroscopic techniques and computational approaches [3]. This technique, based on synchrotron radiation, is non-invasive, non-destructive to a certain extent, very precise and gives the opportunity of measurements at cellular spatial resolution which makes it ideal for biological samples. For these reasons sulphur $K$-edge XANES spectroscopy was performed on prostate cancer cell lines.

Sulphur plays an important role in human body. First of all, it is a part of two amino acids: methionine and cysteine, that builds proteins which are found in every cell in human body. Secondly, it is a part of iron-sulphur $(\mathrm{Fe}-\mathrm{S})$ clusters, essential for numerous biological processes, including mitochondrial respiratory chain activity and various other enzymatic and regulatory functions. Any significant disruptions in $\mathrm{Fe}-\mathrm{S}$ cluster biogenesis and repair are expected to affect numerous basic cellular processes and lead to serious diseases, that are only partially discovered [4]. It is also known that sulphane com- 
pounds (containing two or more sulphur atoms bounded together) have effects in biochemical systems which suggests that they may have natural regulatory functions [5].

Another important sulphur-bearing compound, essential for health, is gluthathion (GSH). It is required for many critical cell processes, including cell differentiation, proliferation, and apoptosis. As a result, any change in GSH homeostasis is implicated in the aetiology and progression of a number of human diseases, including cancer, diseases of ageing and neurodegenerative diseases. GSH deficiency or a decrease in the ratio of GSH/GSSG (glutathione disulfide) manifests itself through the increased susceptibility to oxidative stress. On the other hand, elevated GSH levels, observed in many cancer cells, increase antioxidant capacity and resistance to oxidative stress and that makes cancer cells chemoresistant, which is a major factor that limits drug treatment [6].

However the knowledge about many roles played by sulphur in human body is wide, it has been difficult to describe the processes occurring in cells during carcinogenesis. The present work focuses on the determination and quantification of sulphur forms in prostate cancer cell line PC-3 in comparison with non-cancerous cells.

\section{Materials and methods}

The reference compounds studied included the inorganic compounds: elemental sulphur $\left(\mathrm{S}_{8}\right)$, iron(II) sulphide (FeS), sodium sulphite $\left(\mathrm{Na}_{2} \mathrm{SO}_{3}\right)$, sodium thiosulphate $\left(\mathrm{Na}_{2} \mathrm{~S}_{2} \mathrm{O}_{3}\right)$, barium sulphate $\left(\mathrm{BaSO}_{4}\right)$, copper sulphate $\left(\mathrm{CuSO}_{4}\right)$, ammonium thiosulphate $\left(\left(\mathrm{NH}_{4}\right)_{2} \mathrm{~S}_{2} \mathrm{O}_{3}\right)$ and the organic compounds: bovine albumin, reduced gluthation, L-cysteine, cystine, methionine and oxy-myoglobin. All reference samples were prepared as pellets, obtained from homogeneous powders and mounted on sulphur-free tape. In addition, two types of cell samples were studied: PC-3 cell line, that is derived from advanced androgen independent bone metastasized prostate cancer and PZ-HPV-7, derived from epithelial cells cultured from normal tissue obtained from the peripheral zone of the prostate, used as control. All cells were prepared in accordance with procedure described in Kwiatek et al. [7]. Cultured cells were grown on a support of $1.5 \mu \mathrm{m}$ thick Mylar foil using RPMI-1640 medium (SIGMA-Aldrich) supplemented with $10 \%$ fetal bovine serum (SIGMA-Aldrich), $100 \mathrm{U} / \mathrm{ml}$ penicillin-streptomycin solution (SIGMA-Aldrich), $10 \mathrm{mM}$ HEPES (SIGMA-Aldrich), $1 \mathrm{mM}$ sodium pyruvate (SIGMA-Aldrich) and $4.5 \mathrm{~g} / \mathrm{l}$ glucose. Culture dishes were maintained at $37^{\circ} \mathrm{C}$ in a humidified atmosphere of $5 \% \mathrm{CO}_{2}$. This procedure resulted in monolayer coverage of cells on the Mylar foil.

XANES measurements were performed at the wiggler beamline SUL-X of the synchrotron radiation source ANKA, Karlsruhe (Germany) in fluorescent mode under high vacuum to avoid absorption of the radiation in air. A 7 element $\mathrm{Si}(\mathrm{Li})$ fluorescent detector (Gresham, now $\mathrm{e} 2 \mathrm{v}$ ) was used. The experiments on SUL-X beamline can be done at the energy range $2.14-20 \mathrm{keV}$ with a minimum beam size of $30 \mu \mathrm{m} \times 25 \mu \mathrm{m}$ (horizontal $\times$ vertical) obtainable by focusing with the Kirkpatrick-Baez mirrors and downsizing horizontal and vertical slit gaps. The dimension of the beam spot used for this experiment was $350 \mu \mathrm{m} \times 150 \mu \mathrm{m}$ and $50 \mu \mathrm{m} \times 50 \mu \mathrm{m}$ for reference compounds and for cell samples, respectively. The beam size of $50 \mu \mathrm{m} \times 50 \mu \mathrm{m}$ was chosen for cell samples in order to obtain the best signal to noise ratio and to be close to cellular spatial resolution. The energy was scanned with the double crystal monochromator (Si(111) crystal) in steps of $5 \mathrm{eV}, 2 \mathrm{eV}$ and $0.2 \mathrm{eV}$ in the region of -100 to $-50 \mathrm{eV}$ before the edge, -50 to $-20 \mathrm{eV}$ before the edge and in the edge region, respectively. Higher harmonics has been suppressed by detuning the rocking curve to about $50 \%$ of its maximum intensity. Energy has been calibrated to $2481.4 \mathrm{eV}$ at the maximum of the sulphate white line of a scotch tape.

\section{Results and discussion}

\subsection{Determination of oxidation states of sulphur}

All the presented XANES spectra were baseline corrected and normalized in Athena code [8].

The main and most important information one can get from the spectra of reference compounds is the relation between the energy of the white line maximum and the oxidation state of sulphur. Since sulphur occurs in various oxidation states and the difference in the energy of the white line maximum in XANES spectra may differ by about $11 \mathrm{eV}$, it is clear that sulphur XANES spectroscopy has great potential for determining sulphur speciation [9].

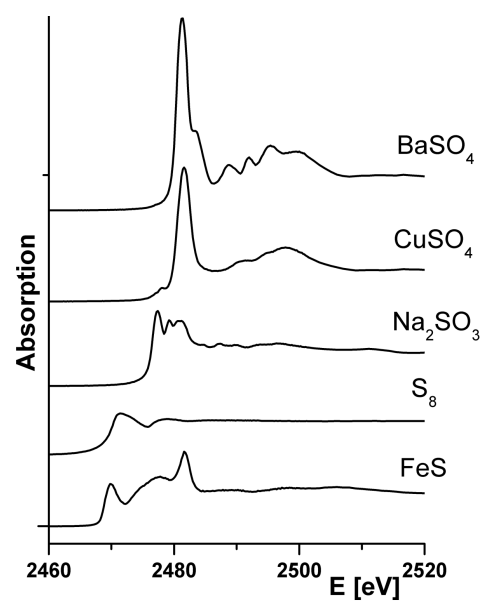

Fig. 1. Sulphur $K$-edge XANES spectra of the five reference compounds: $\mathrm{FeS}, \mathrm{S}_{8}, \mathrm{Na}_{2} \mathrm{SO}_{3}, \mathrm{CuSO}_{4}$, and $\mathrm{BaSO}_{4}$.

Figure 1 shows the XANES spectra of five reference compounds: FeS, $\mathrm{S}_{8}, \mathrm{Na}_{2} \mathrm{SO}_{3}, \mathrm{CuSO}_{4}$, and $\mathrm{BaSO}_{4}$, representing sulphur's various oxidation states, and Fig. 2 presents the relation between the energy of the white line maximum and the oxidation state of sulphur in these 


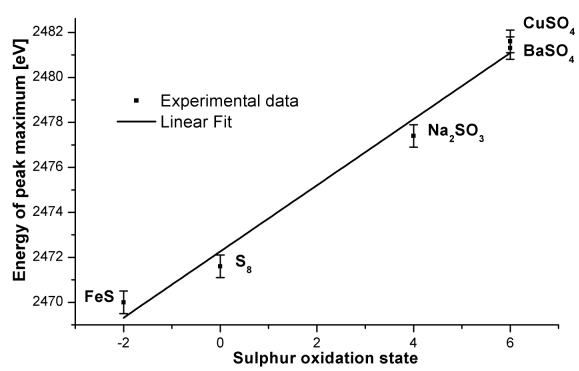

Fig. 2. The relation between sulphur oxidation states and the energy of white line maximum in $\mathrm{S} K$-edge XANES measurements. Experimental data were fitted with least-squares line.

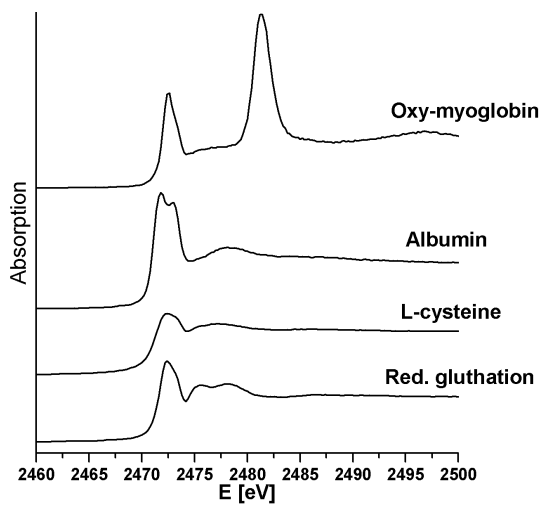

Fig. 3. Sulphur $K$-edge XANES spectra of the four organic reference compounds: reduced gluthation, L-cysteine, albumin and oxy-myoglobin.

compounds. The linear fit to the experimental points was made and the obtained regression equation was used to calculate unknown oxidation states. First, the oxidation states for thiosulphates and organic reference compounds were established.

Figure 3 shows the spectra of selected organic reference compounds, where one can notice that the position of the white line is similar for all compounds (except the second peak of the oxy-myoglobin, that comes from sulphate ligand). The results, presented in Table I, are consistent with the literature [9-12]. As should be noticed, there is a difference between formal and apparent oxidation state, received from XANES measurements, for example the apparent oxidation states of sulphur in thiosulphates, obtained from XANES data, are -1 and +5 , while the formal ones are -2 and +6 . Such a difference could occur due to calibration curve which has been done on the basis of chemical compounds in which there are ionic bonds which is not true in case of thiosulphates.

The worked out procedure was used to establish the speciation of sulphur in cell samples.

\subsection{Quantitative results}

In order to establish what speciation of sulphur occurs in cells the procedure of deconvolution of XANES spec-
TABLE I

The oxidation states of sulphur in thiosulphates and organic reference compounds calculated using the regression equation.

\begin{tabular}{c|c|c}
\hline \hline Compound's name & $\begin{array}{c}\text { Energy of white line } \\
\text { maximum [eV] }( \pm 0.5 \mathrm{eV})\end{array}$ & $\begin{array}{c}\text { Calculated } \\
\text { oxidation state }\end{array}$ \\
\hline \multicolumn{3}{c}{ Thiosulphates } \\
\hline$\left(\mathrm{NH}_{4}\right)_{2} \mathrm{~S}_{2} \mathrm{O}_{3}$ & 2470.9 & $-0.9 \pm 0.2$ \\
$\mathrm{Na}_{2} \mathrm{~S}_{2} \mathrm{O}_{3}$ & 2479.8 & $5.1 \pm 0.3$ \\
\multicolumn{2}{c}{ Organic reference compounds } \\
\hline albumin & 2470.9 & $-0.9 \pm 0.2$ \\
reduced gluthation & 2472.5 & $-0.2 \pm 0.2$ \\
L-cysteine & 2472.5 & $0.2 \pm 0.2$ \\
cystine & 2471.6 & $0.2 \pm 0.2$ \\
methionine & 2472.5 & $-0.4 \pm 0.2$ \\
oxy-myoglobin & 2472.6 & $0.2 \pm 0.2$ \\
& 2481.3 & $6.2 \pm 0.3$
\end{tabular}

tra was used. From every sample spectra were collected in four different points and these four spectra were deconvoluted individually, and then the average for each sample was calculated. The deconvolution was made in Athena code and experimental spectra were fitted with two Gaussians convoluted with the arctangent shape of X-ray edge. All parameters of the fit were loosely constrained, except the FWHM of the second Gaussian, additionally several sets of initial parameters were tested and they all gave reproducible results.

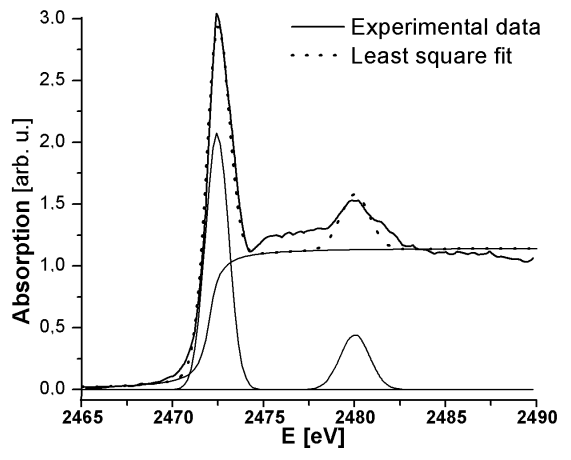

Fig. 4. The experimental S $K$-edge XANES spectrum of PC-3 cells fitted with two Gaussian peaks and one arctangent function.

The representative deconvoluted spectrum is presented in Fig. 4. The number of Gaussian peaks were chosen after short analysis of the spectra. In samples with unknown number of sulphur species the main question is how to differ the fine structure of the spectrum from the absorption edges. Whereas there are no problems in the identification of the first absorption edge, there are no clear criterions that let to analyse the rest of the spectrum. This problem is well illustrated in Fig. 1, on the spectrum of $\mathrm{BaSO}_{4}$, where above the white line there are few other oscillations characteristic for a typical XANES 
structures and there is only one sulphur form. In this paper the criterion of asymmetry of the absorption edge was used and in first approach only two sharp peaks, that fulfil this condition, were taken into account. The problem of the analysis and algorithm of identification will be a subject of further studies.

Two identified peaks are representative of the functional groups of sulphur which are likely to be present in biological system: thiols (for example cysteine, cystine and gluthatione) and highly oxidized sulphur as in sulphonates and sulphates. Every Gaussian represents the transition of the electron from $s$ to $p$ state and arctangent step function is related with the transition of the ejected photoelectrons into the continuum. In every spectrum, the area of each Gaussian curve was calculated and obtained results are presented in Table II. Additionally, the positions of fitted Gaussian peaks were used to compute the oxidation state of each sulphur form and results are presented in Table III.

The area under Gaussian peaks, obtained after deconvolution of XANES spectra of cell samples.

\begin{tabular}{c|c|c|c|c|c|c}
\hline \hline \multirow{2}{*}{$\begin{array}{c}\text { Energy of Gaussian maximum } \\
{[\mathrm{eV}]( \pm 0.5 \mathrm{eV})}\end{array}$} & \multicolumn{3}{|c|}{ PC-3 cells } & \multicolumn{3}{|c}{ PZ-HPV-7 cells (control) } \\
\cline { 2 - 6 } & Sample 1 & Sample 2 & Average & Sample 1 & Sample 2 & Average \\
\hline 2472.4 & $3.00 \pm 0.04$ & $3.24 \pm 0.04$ & $3.12 \pm 0.03$ & $3.18 \pm 0.03$ & $3.12 \pm 0.03$ & $3.15 \pm 0.02$ \\
2480 & $1.50 \pm 0.03$ & $1.17 \pm 0.04$ & $1.34 \pm 0.02$ & $1.26 \pm 0.03$ & $1.37 \pm 0.03$ & $1.31 \pm 0.02$ \\
\cline { 4 - 6 } & & & & & &
\end{tabular}

TABLE III

The oxidation states of sulphur in cell samples calculated using the regression equation.

\begin{tabular}{c|c}
\hline \hline $\begin{array}{c}\text { Energy of Gaussian maximum } \\
{[\mathrm{eV}]( \pm 0.5 \mathrm{eV})}\end{array}$ & $\begin{array}{c}\text { Calculated oxidation } \\
\text { state }\end{array}$ \\
\hline 2472.4 & $0.1 \pm 0.2$ \\
2480 & $5.3 \pm 0.3$
\end{tabular}

The results show that the sulphur $K$-edge XANES spectra of both cancerous and non-cancerous cells could be reproduced by two Gaussian peaks (two model compounds). The fraction of reduced sulphur (with apparent oxidation state around 0) is comparable in both cancerous and non-cancerous cells. There are also no significant changes in oxidized species. Nevertheless, the dispersion of the results obtained for the cancerous cells is worth to highlight and could indicate that some sort of reduction or oxidation process, caused by oxidation stress, occurred in these cells. Due to the small number of samples and measured spectra the experiment should be continued and evidenced by studies on other available prostate cancer cell lines.

The presented procedure was earlier exploited mainly in geochemical studies [13]. There were also works presenting the analysis of chemical forms of sulphur in simple biological systems, with the use of the model compounds spectra, that requires very precise preparation of reference samples and careful analysis $[14,15]$.

\section{Conclusions}

The biochemical processes occurring in cells during the development and progression of prostate cancer remains largely undefined. Our studies have focused on the role of sulphur in cancer transformation in prostatic cells, with the use of modern and very sensitive synchrotron methods.

Two kinds of samples, with prostate cancer PC-3 cell line and with non-cancerous PZ-HPV-7, were analysed with X-ray absorption near edge structure spectroscopy. With the use of reference compounds, the relation between the energy of XANES white line maximum and the oxidation state was obtained. The obtained results showed the differences between formal and apparent oxidation state, received from XANES measurements, for example the apparent oxidation states of sulphur in thiosulphates, obtained from XANES data, are -1 and +5 , while the formal ones are -2 and +6 . Afterwards, $K$ -edge sulphur XANES spectra of cell samples were deconvoluted with two Gaussian peaks, representing model compounds that are present in human cells. The presented results show no significant differences in the fraction of reduced and highly oxidized sulphur between cancerous and non-cancerous cells, but there are changes between single samples in the case of cancerous cells. This may indicate changes in redox balance due to the oxidative stress. The most important result of these studies is the procedure that lets to analyse the XANES spectra of biological material and this can be helpful in better understanding on the mechanism of carcinogenesis and can benefit in both diagnostic and cancer treatment. Nevertheless, further studies with different prostate cancer cell lines are required.

\section{Acknowledgments}

The research leading to these results has received funding from European Community's Seventh Framework Programme (FP7/2007-2013) under grant agreement no. 226716. We acknowledge ANKA for granting beamtime in the proposal OTH-72. 


\section{References}

[1] W.M. Kwiatek, M. Podgórczyk, C. Paluszkiewicz, A. Balerna, A. Kisiel, Acta Phys. Pol. A 114, 463 (2008).

[2] B. Ravel, M. Newville, J. Synchrotron Rad. 12, 537 (2005).

[3] WHO World Cancer Report 2008, Eds. P. Boyl B. Levin, Lyon 2008.

[4] S.M. Dhanasekaran, T.R. Barrette, D. Ghosh, R. Shah, S. Varambally, K. Kurachi, K.J. Pienta, M.A. Rubin, A.M. Chinnaiyan, Nature 412, 822 (2001).

[5] R.W. Strange, M.C. Feiters, Curr. Opin. Struct. Biol. 18, 609 (2008)

[6] T.A. Rouault, W.H. Tong, Trends Genet. 24, 398 (2008).

[7] J.I. Toohey, Biochem. J. 264, 625 (1989).

[8] N. Ballatori, S.M. Krance, S. Notenboom, S. Shi, K. Tieu, C.L. Hammond, Biol. Chem. 390, 191 (2009).
[9] G.S. Waldo, R.M.K. Carlson, J.M. Moldowan, K.E. Peters, J.E. Penner-Hahn, Geochim. Cosmochim. Acta 55, 801 (1991).

[10] A. Vairavamurthy, B. Manowitz, G.W. Luther, III, Y. Jeon, Geochim. Cosmochim. Acta 57, 1619 (1993).

[11] M.J. Morra, S.E. Fendorf, P.D. Brown, Geochim. Cosmochim. Acta 61, 683 (1997).

[12] C. Jacob, G.I. Giles, N.M. Giles, H. Sies, Angew. Chem. Int. Ed. 42, 4742 (2003).

[13] T. Schäfer, G. Buckau, R. Artinger, J.I. Kim, S. Geyer, M. Wolf, W.F. Bleam, S. Wirick, C. Jacobsen, Organic Geochem. 36, 567 (2005).

[14] I.J. Pickering, R.C. Prince, T. Divers, G.N. George, FEBS Lett. 441, 11 (1998).

[15] G. Almkvist, K. Boye, I. Persson, J. Synchr. Radiat. 17, 683 (2010). 